\title{
La medición de la Cooperación Sur-Sur colombiana a cuarenta años del Paba: Una propuesta de convergencia entre cuantificación y agregación de valor
}

Fernando Nivia-Ruiz ${ }^{*}$

\section{RESUMEN}

Transcurridos cuarenta años desde el Plan de Acción de Buenos Aires y luego de celebrarse Ia II Conferencia de Naciones Unidas sobre la Cooperación Sur-Sur, Paba+40, la necesidad de contar con información, cifras y estadísticas sobre esta modalidad de cooperación se encuentra aún vigente. Actualmente, existe un amplio debate académico al respecto que aquí se expone como marco general, para luego presentar y analizar en detalle las últimas cifras e información sobre la css colombiana y, en particular, su reciente propuesta de medición denominada Modelo de Cuantificación y Agregación de Valor, utilizando una iniciativa de css colombo-boliviana recientemente ejecutada, que evidencia la importancia del modelo, así como sus retos y desafíos.

Palabras clave: Cooperación Sur-Sur, medición, cuantificación, agregación de valor.

* Especialista en cooperación internacional para el desarrollo y candidato a magíster en políticas públicas para el desarrollo. Investigador del Centro de Investigación en Cooperación Internacional y Desarrollo-Universidad Nacional de San Martín (Cicid-Unsam), (Argentina). [fernandoniviaruiz@gmail.com], [https://orcid.org/0000-0003-42270730].

Recibido: 31 de mayo de 2019 / Modificado: 24 de julio de 2019 / Aceptado: 26 de julio de 2019

Para citar este artículo:

Nivia-Ruiz, F. (2020). La medición de la Cooperación Sur-Sur colombiana a cuarenta años del Paba: Una propuesta de convergencia entre cuantificación y agregación de valor. OASIS, 31, pp. 51-72

Dor: https://doi.org/10.18601/16577558.n31.05 
The measurement of Colombian South-South Cooperation forty years after BAPA: Proposal of convergence between quantification and value addition

\section{ABSTRACT}

Over forty years since the Buenos Aires Plan of Action, and after the $2^{\text {nd }}$ United Nations Conference on South-South Cooperation, $\mathrm{BAPA}+40$, the need to have information, data and statistics about this cooperation modality continues to be in force. At this time, there is an ongoing and broad academic discussion introduced here as a general framework, followed by a detailed analysis of the latest Colombian ssc data and information, and particularly its recent proposal of a measurement approach named Quantification and $V a$ lue Addition Model, seen through the recently implemented Colombo-Bolivian initiative of ssc which exemplies the model's relevance and challenges.

Key word: South-South Cooperation, measurement, quantification, value addition.

\section{INTRODUCCIÓN}

El 22 de marzo de 2019, el canciller argentino Jorge Faurie dio por culminada la II Conferen- cia de Alto Nivel de las Naciones Unidas sobre la Cooperación Sur-Sur (css), conocida como Paba+40. Más de 150 países estuvieron reunidos durante tres días, nuevamente en Buenos Aires, junto a una innumerable cantidad de organizaciones de la sociedad civil, academia y sector privado. Allí se aprobó por unanimidad el documento final con 37 puntos en el marco de hondas gratitudes, muestras de aprecio y beneplácito.

Esta II Conferencia, así como la primera y los foros de diálogo político, cada vez más frecuentes sobre la css, han posibilitado el ingreso a la agenda de una pluralidad de temas como medio ambiente, migraciones, género, entre otros. De igual forma, es evidente la vigencia de los debates que se sostienen en los países del Sur Global ${ }^{1}$, respecto a la necesidad de generar y contar con evidencias precisas y concretas a partir de la implementación de una política de cooperación cada vez más extendida entre los países y regiones en desarrollo.

Iberoamérica, particularmente, ha adquirido un liderazgo importante en la generación de información respecto a los principales flujos de css bilateral, triangular y regional entre los países miembros del Programa Iberoamericano para el Fortalecimiento de la Cooperación Sur-Sur (PIFCSS), publicando un informe anual que da cuenta de más de 7.300 iniciativas entre 2006 y 2015, reflejo de la extensa

\footnotetext{
Este concepto se entiende según lo definido por Ayllón et al. (2013), como aquella designación simbólica para denominar a una amplia gama de naciones en desarrollo, diversas por sus historias, orígenes y tradiciones, con múltiples enfoques en lo relativo al poder, la cultura o la identidad.
} 
tradición de cooperación técnica que acumula la región (Segib, 2018b).

La discusión actual sobre las cifras, datos, información y resultados de la css, se encuentra abierta y mucho más enriquecida en comparación con la década del Paba. Cuantificar, monetizar o valorar la css son conceptos utilizados muchas veces de forma indistinta e incluso con el objetivo de significar cuestiones diferentes. Es por esto que distintos países, incluido Colombia, han optado por desarrollar sus propias herramientas metodológicas que les permitan realizar mediciones sobre la css que ofrecen, conforme con sus capacidades humanas, técnicas y financieras.

En este sentido, el presente artículo se estructura en cinco secciones. En primer lugar presenta un panorama muy general sobre las principales referencias de contexto del Paba y el Paba+40, principalmente respecto a la generación de información en la css. En segundo término, introduce el debate vigente sobre la cuantificación de la css, presentando los principales argumentos y avances -aún no definitivos- que existen al respecto. Una tercera sección analiza el caso colombiano y su trayectoria en la generación de información sobre css hasta llegar a su modelo actual de cuantificación, junto a la introducción del concepto de la agregación de valor como medio para valorizar su css. En cuarto lugar, se analiza el citado modelo a la luz de un caso concreto de css implementado por la Agencia Presidencial de Cooperación Internacional de Colombia (APC-Colombia), para dar paso a las conclusiones y reflexiones finales.

\section{PABA Y PABA+40: LA INFORMACIÓN DE LA CSS}

El Paba ha sido reconocido históricamente como el hito fundacional de la CTPD en donde se aprobaron una serie de recomendaciones para promover esta modalidad de cooperación en una coyuntura histórica muy particular (la del Movimiento de Países No Alineados, el Nuevo Orden Económico Internacional y la descolonización) y con unas tendencias que ya señalaba Tomassini (1976), influirían la adopción de nuevas formas de cooperación entre países en desarrollo, disminuyendo su grado de dependencia externa y estimulando sus relaciones recíprocas.

Para los países de la región latinoamericana no eran años sencillos. Aunado a la crisis petrolera de 1973, buena parte de estos vivían períodos de dictaduras y la cooperación no era parte integrante de sus políticas públicas (Lazo, 2018). Tampoco lo fue la década siguiente con la crisis de la deuda, pues a pesar de la relevancia que se pudiera dar a la cooperación horizontal, la tendencia declinante de los montos financieros asignados a esta, constituía el principal obstáculo para avanzar en su consolidación (Ilpes, 1984).

Es innegable que la cooperación entre países en desarrollo, discutida en marzo de 2019, dista mucho de la cooperación moldeada en septiembre de 1978. El contexto internacional y del mundo en desarrollo ha cambiado profundamente. No obstante, cuestiones fundacionales como el interés por contar con información confiable sobre la css se mantienen vigentes luego de cuarenta años (ver Tabla 1). De allí que a lo largo de estas 
Tabla 1

Elementos presentes en el Paba y Paba+40

\begin{tabular}{|c|c|c|}
\hline & Conferencia sobre CTPD (1978) & Conferencia sobre css (2019) \\
\hline Contexto internacional & $\begin{array}{l}\text { - Distensión de la guerra fría. } \\
\text { - Declive de la hegemonía de EE.uU. } \\
\text { - Proyecto político del Tercer Mundo. }\end{array}$ & $\begin{array}{l}\text { - Orden multipolar. } \\
\text { - Crisis del multilateralismo. }\end{array}$ \\
\hline Contexto del Sur Global & $\begin{array}{l}\text { - Auge del proyecto Sur (Bandung). } \\
\text { - Conclusión del proceso de descoloniza- } \\
\text { ción (MNOAL). }\end{array}$ & $\begin{array}{l}\text { - Países del Sur ahora potencias (China). } \\
\text { - Alta participación del Sur en la econo- } \\
\text { mía global. }\end{array}$ \\
\hline Paradigma vigente & Teoría de la dependencia - Noei. & Agenda 2030 y oDs. \\
\hline Concepto CTPD/css & $\begin{array}{l}\text { Proceso multidimensional. Bilateral o } \\
\text { multilateral. Con carácter, subregional, } \\
\text { regional o interregional. Organizada } \\
\text { por los gobiernos entre sí. Cooperación } \\
\text { técnica. }\end{array}$ & $\begin{array}{l}\text { Realizada entre países del Sur. En esferas } \\
\text { económica, social, cultural, ambiental } \\
\text { y técnica, entre otras. Tiene lugar en } \\
\text { contextos bilaterales, regionales o } \\
\text { interregionales. }\end{array}$ \\
\hline Decisiones & Paba. (9 objetivos y 21 recomendaciones.) & $\begin{array}{l}\text { Documento final conciso y específico } \\
\text { con visión de futuro (A/RES/71/318). }\end{array}$ \\
\hline
\end{tabular}

Fuente: Elaboración propia, 2019 a partir de Surasky y Ayllón (2018).

décadas algunas regiones y países del Sur, incluido ahora Colombia, hayan decidido iniciar la implementación de acciones principalmente gubernamentales al respecto.

Pese a las marcadas diferencias que existen entre ambos escenarios, es posible identificar en ellos las referencias que en su momento señalaron sobre la importancia de los datos e información de la CTPD, hoy css. El Paba en su recomendación No. 4 abogaba por el fortalecimiento de los sistemas nacionales de información en materia de CTPD, y hacía lo propio respecto al mejoramiento de la información para ampliar el alcance y aprovechamiento de la misma, en la recomendación No. 20. Incluso, dentro de sus medidas subregionales y regionales proponía normas relativas a la corriente de informaciones sobre la CTPD.
Décadas más tarde, el Paba+40 alienta a los países a elaborar sistemas propios para evaluar la calidad y los efectos de sus programas de css y ст, y a que sean apoyadas iniciativas de recopilación, coordinación, difusión de información, de datos y de evaluación de la css. Igualmente, insta a mejorar la recopilación de datos con el fin de promover la cooperación en la elaboración de metodologías y estadísticas (párrafo 25).

Si bien, en el Paba la generación de información estaba más abocada hacia la identificación de necesidades, capacidades y experiencias de los países del Sur para el uso de la CTPD, claramente el Paba +40 da un salto sustancial al indagar por información, cifras y metodologías que permitan dar cuenta, más bien, de los 
efectos, resultados y ejercicios que posibiliten la evaluación de la css.

En uno u otro sentido, en Latinoamérica es posible identificar avances significativos. Si se trata de generar información sobre experiencias y fortalezas de los países para la css, la práctica más utilizada han sido los catálogos o inventarios que recogen las capacidades técnicas de estos. Argentina (en 2015), Colombia $(2016)^{2}$, El Salvador (2016), México (2018) y Uruguay (2019), son solo algunos ejemplos recientes de ello.

Ahora bien, en términos de instrumentos y metodologías de cuantificación, el universo parece estar reducido a aquellos países que cuentan con mayores capacidades nacionales e incorporan este tipo de mediciones. Los casos de Brasil, Chile y México se encuentran detallados en el Documento de Trabajo del PIFCSs (2016) que da cuenta de las propuestas metodológicas realizadas por estos.

A cuatro décadas del Paba aún persisten los esfuerzos que buscan generar información y ahondar en la cuantificación de la css en un buen número de países y organismos regionales. Recientemente, Costa Rica presentó su metodología para cuantificar los aportes del sector público en los proyectos de cooperación que ejecutan, incluida la css (Mideplan, 2018). Colombia objeto de estudio del presente escrito, viene construyendo su propio camino con propósitos aún mayores.

\section{CUANTIFICACIÓN DE LA CSS: DEBATE ABIERTO E INCONCLUSO}

La importancia y vigencia de la css parece no estar más en discusión. La Agenda 2030 y, en particular el oDs 17, la incorporan dentro del propósito de no dejar a nadie atrás. El hecho es que los países realizan la css, según Ayllón (2015), basados en los mismos principios, pero con diferentes instrumentos, modalidades, recursos, amplitud y profundidad. Esto implica que aún co-existen múltiples aproximaciones a la css, lo cual constituye un inmenso desafío para obtener información comparable sobre su alcance, extensión, nivel de implementación (Yu, 2019) y para abordar su cuantificación.

Dado que el espacio no permite ahondar en la discusión epistemológica de la css, vale la pena acotar lo señalado recientemente por Bracho (2018) y Besharati (2019) ${ }^{3}$ quienes sostienen que existen dos comprensiones básicas sobre css: la primera, denominada la css del "espiritu de Buenos Aires", que se circunscribe a la cooperación técnica, principalmente a través del envío de expertos como es practicada en la mayoría de países latinoamericanos; y la del "espiritu de Bandung" que trasciende a la primera y enfatiza en la cooperación económica, financiera y comercial, típica en regiones como Asia. En este marco conceptual surgen los debates vigentes sobre la cuantificación de la css.

\footnotetext{
2 El portafolio de experiencias nacionales y territoriales documentadas que son la base de la css que el país ofrece se denomina Saber Hacer Colombia.

3 Intervención en el Side Event del Paba+40: "La CSS a 40 años del Paba", organizado por la UCM y la UBA. 19 de marzo de 2019. Buenos Aires.
}

OASIS, ISSN: 1657-7558, E-ISSN: 2346-2132, N³1, Enero-Junio de 2020, pp. 51-72 
Como punto de partida se coincide en la escasa disponibilidad de datos, líneas de base, así como disímiles capacidades para generar información sobre css (Huitrón, 2016; PNUD, 2016; Xalma, 2013; Brics Policy Center, 2017) y en cómo las estadísticas han sido limitadas, inadecuadas, incompletas o no están disponibles (Besherati y MacFeely, 2019), vacío que supondrá siempre un obstáculo para conocer, defender y evaluar los logros y aportes de la css (Surasky, 2018). Aún se discute el plano preponderante (político, financiero o técnico) en dónde ubicar las principales contribuciones de la css; dónde centrar los criterios y parámetros de su medición; así como la viabilidad de contar con una o diversas metodologías de cuantificación.

Adicionalmente, se evidencia la falta de consenso sobre qué y cómo medir la css, por lo cual este escrito acoge el planteamiento de Pérez y Huitrón (2018) quienes ubican tres enfoques que se sintetizan, al máximo, en la Tabla 2.

Respecto al primer referente de análisis, Lopes (2017) advierte los riesgos de medir la Css únicamente en términos monetarios, pues generaría implicaciones negativas para la política exterior de los países, ya que usar la css únicamente como un mecanismo complementario de financiación subestima su dimensión política ${ }^{4}$ y aboga por un modelo innovador centrado en evaluar la coherencia entre todos los diferentes aportes y beneficios reales derivados de esta.

DiCiommo (2017) llama la atención también sobre la monetización de la css, considerando que los recursos no financieros siempre tendrán un costo menor en los países en desarrollo, y aporta a la discusión tres posibles niveles de aproximación a la cuantificación de la css.

El primero de ellos, es el nivel de las experiencias nacionales de países como Brasil, Colombia, Turquía o Emiratos Árabes que generan y reportan información cuantitativa y cualitativa. Un segundo nivel, son las experiencias regionales como el caso de la Segib con el Informe de la css ya mencionado, y la Cepal en la definición de una metodología para medir la $\mathrm{CSs}^{5}$; y por último, las experiencias internacionales como la del Network of Southern Think Tanks (Nest) que propone criterios, métodos e indicadores de medición de la $\operatorname{css}^{6}$ asociados al segundo enfoque de evaluación de procesos arriba indicado.

Respecto a los indicadores para la css, el PIFCSS inició en 2011 un proceso de construcción intergubernamental advirtiendo que en medio de criterios y restricciones, podría generarse una batería potencialmente muy

\footnotetext{
4 Huitrón (2016) señala, por otra parte, que la inquietud por conocer las ventajas reales que supone la css en el desarrollo más allá del vínculo político e ideológico, supuso plantear la hipótesis de que la css puede aportar nuevos mecanismos y acciones de cooperación.

5 Cepal realizó, en 2016, una propuesta de medición para la css a través de una Cuenta Satélite en el marco del Sistema de Cuentas Nacionales.

6 Desarrollo completo en Nest (2015) Developing a conceptual framework for ssc. Working Document. September.
} 
Tabla 2

\section{Líneas de acción y discusión sobre cuantificación de la css}

\begin{tabular}{|l|l|l|l|}
\hline \multicolumn{1}{|c|}{ Enfoques } & \multicolumn{1}{|c|}{ Fundamentos } & \multicolumn{1}{c|}{ Medición } & \multicolumn{1}{c|}{ Experiencias } \\
\hline Cuantificación & Monetización de la css & $\begin{array}{l}\text { Costos directos/indirectos, horas } \\
\text { experto, contribuciones financieras, } \\
\text { ayuda humanitaria, etc. }\end{array}$ & $\begin{array}{l}\text { México } \\
\text { Chile } \\
\text { Turquía } \\
\text { China, etc. }\end{array}$ \\
\hline Calidad & $\begin{array}{l}\text { Evaluación de procesos } \\
\text { cualitativos de gestión (no de } \\
\text { resultados) y asociación de } \\
\text { relaciones de css entre países }\end{array}$ & $\begin{array}{l}\text { Cumplimiento de los principios } \\
\text { constitutivos de la css (horizontalidad, } \\
\text { equidad, reciprocidad, etc.) }\end{array}$ & Brics \\
\hline Valorización & $\begin{array}{l}\text { Resultados y posible impacto } \\
\text { de la css, más allá de la } \\
\text { monetización }\end{array}$ & $\begin{array}{l}\text { Resultados y beneficios obtenidos } \\
\text { de las iniciativas de css, revelando } \\
\text { las insuficiencias de los dos enfoques } \\
\text { anteriores }\end{array}$ & Colombia \\
\hline
\end{tabular}

Fuente: Elaboración propia, 2019, a partir de Pérez y Huitrón (2018).

amplia en donde el reto ineludible pasaba por mejorar las capacidades de registro y reporte de los países (PIFCss, 2013). A la fecha existe el Sistema Integrado de Datos de Iberoamérica sobre css, en donde se registra la información asociada a los proyectos, iniciativas y sectores de la css Iberoamericana.

El debate, como se advertía, aún se encuentra abierto y en un escenario de Paba +40 y a futuro, Besherati y MacFeely (2019) consideran abiertos tres posibles escenarios en términos de información y medición de la css. El del statu quo donde los datos, incluso de la css, continúen siendo elaborados por la Ocde ${ }^{7}$ y los países del norte. La segunda opción del mini- mum common denominators, donde los países del Sur traten de armonizar sus reportes básicos de cooperación; o un último y nuevo camino hacia la new measure que implicará acuerdos en una definición holística de la css con claros parámetros estadísticos para su cuantificación; lo cual parece encontrarse en un mediano o largo término a la luz de las evidencias actuales.

En medio de esta inspiradora cuestión, Colombia ha decidido iniciar una senda que incorpora tanto la cuantificación ya planteada, como la valorización de su css. Este caso puede entenderse como un ejercicio que intenta ir más allá de una metodología de contabilización, centrándose en los resultados y el aporte

$7 \quad$ La Ocde (2018) calculó el monto de cooperación brindada por Brasil en us $\$ 316$ millones (para 2013); Colombia en us $\$ 44$ millones (para 2016) y Chile en us $\$ 33$ millones (para 2016). Igualmente, Naciones Unidas (2018) estimó las aportaciones a la css en más de us $\$ 20.000$ millones para 2013. 
que esta cooperación brinda en términos de capital humano y, en última instancia, al desarrollo (Pérez y Huitrón, 2018).

\section{CSS COLOMBIANA: EN BÚSQUEDA DE LOS DATOS E INFORMACIÓN}

En la década de los años sesenta, el país conformó la primera oficina encargada de gestionar la cooperación internacional. La Unidad de Proyectos Especiales y Crédito Externo fue creada en 1968, pasando a convertirse en la División Especial de Cooperación Técnica (Decti), con un grupo encargado de liderar la CTPD, aunque sin los medios necesarios para implementar este tipo de iniciativas (Bergamaschi et al., 2017b). De hecho, solo hasta la década de los años ochenta se elaboró la primera base de datos con información de los programas y proyectos de cooperación en el país (Acción Social, 2008).

Es a finales de esta década donde se encuentran los orígenes y primeros datos de la entonces CTPD colombiana, realizada a través de organismos regionales con países centroamericanos y caribeños, e incluso se registran las primeras acciones de Ст (Guáqueta, 1995; Henao 1991; DNP, 1995). Estos son también los años en que se creó el Fondo de Cooperación para Centroamérica y el Caribe, mediante Decreto No. 2157/1982, siendo la primera cuenta que permitía disponer recursos del presupuesto nacional para realizar acciones de oferta de CтрD (Sanín, 2010).

Años más tarde, el Documento Conpes 2768/1995 "Política Nacional de Cooperación Internacional" propuso, entre otros asuntos, fortalecer la institucionalidad de la cooperación en el país, con lo cual se crearon la Agencia Colombiana de Cooperación Internacional (Acci) y el Fondo de Cooperación y Asistencia Internacional (Focai) mediante la Ley 318/1996 ${ }^{8}$. A partir de entonces, y aún con algunas dificultades, se empezaron a generar datos más frecuentes sobre la AOD y, en alguna medida, sobre la css colombiana?

El inicio del siglo xx dio cuenta del nuevo Sistema de Monitoreo y Evaluación de Proyectos (Simep) como respuesta a la necesidad de cualificar los datos relevantes y la rendición de cuentas (Acci, 2002). Los informes de la agencia empezaron a presentar información sobre iniciativas, socios y financiación de la Css colombiana ${ }^{10}$, aunque con indicadores

\footnotetext{
8 Deroga el Decreto 2157/1982.

9 El Conpes 2968/1997 proponía incluso que Colombia liderara e hiciera parte de la creación de un sistema de información regional de CTPD para fortalecer y coordinar las acciones de cooperación horizontal con Centroamérica y el Caribe.

10 Entre junio de 2001 y mayo de 2002 se relacionan seis programas de cooperación técnica vigentes. Se precisa que la financiación de la CTPD se realizaba a través del Focai (exclusivamente para la oferta de cooperación), de la oIM (para actividades de demandas de experiencias internacionales) y de recursos de contrapartida de entidades nacionales. Durante dicho período Colombia ejecutó us\$179.150 en CTPD.
} 
siempre diferentes y datos poco consistentes (Ramírez, 2005).

Posteriormente, se creó el Sistema de Información de la Ayuda Oficial al Desarrollo (Siaod) que concentraba los esfuerzos de registro, únicamente, en los montos de cooperación que recibía el país; ello desagregado por municipios y áreas beneficiarias (Acción Social, 2005). Se contaba con un mapa de cooperación ${ }^{11}$ administrado por la entonces Agencia para la Acción Social y la Cooperación Internacional (Acción Social) que en 2005 reemplazó a la Acci, y permitía visualizar las actividades ejecutadas a través de programas de css (Nivia-Ruiz, 2009).

En APC-Colombia, la Agencia en funciones desde 2011, el seguimiento a la Css у ст se realiza a través de dos instrumentos: Una matriz de seguimiento y un cuadro de programación y ejecución de iniciativas que incluye países, regiones, beneficiarios, sectores (APC-Colombia, 2015b) ambas desarrolladas en hojas de cálculo de Microsoft Excel. A la fecha, la Agencia viene implementando un nuevo sistema de información para el seguimiento de la cooperación llamado ahora Cíclope el cual se encuentra en un $90 \%$ de avance (APC-Colombia, 2019a) e incluye un geovisor (nueva versión del mapa de cooperación) ${ }^{12}$. Actualmente, también existe una política de datos abiertos sobre la AOD y la css del país.
En síntesis, tres elementos pueden abstraerse de este recorrido. Primero, se evidencia cómo los recurrentes cambios en la institucionalidad de la cooperación, generan múltiples modificaciones en los sistemas de información utilizados para el reporte y seguimiento respectivo. Por otra parte, es claro que estos sistemas han tenido una fuerte orientación hacia el registro y seguimiento de la AOD, principalmente.

Por último, resulta nada sencillo trazar líneas uniformes sobre la información de la css publicada, toda vez que no siempre se utilizan o reportan las mismas variables o unidades de registro. Lo anterior deja en evidencia los retos que ya advertía Henao (1991) tiene el país en materia de información, registro y reporte de su cooperación, fundamentalmente css, pese al camino recorrido hasta la fecha.

\subsection{DATOS Y CIFRAS RECIENTES DE LA CSS COLOMBIANA}

En la actualidad, no parece haber duda de que la fuente de información más estandarizada que existe en la región sobre css es el Informe de la Segib publicado anualmente, aunque con cifras de dos años atrás; sin embargo, es a la fecha un buen termómetro de la css Iberoamericana. Contiene información cada vez más precisa y especializada sobre css

11 Experiencia innovadora compartida por Colombia con varios países y agencias de cooperación de la región. Además, incluida dentro de las 110 historias de caso de la css elaborado por el entonces Task Team de la css (GT-CSS, 2010).

$12 \mathrm{Al}$ momento de elaborar este escrito únicamente reporta iniciativas de AOD. 
bilateral, regional y triangular (Prado, 2016). El panorama reciente del caso colombiano se aprecia en la Figura 1.

Adicionalmente, según los informes publicados por APC-Colombia, el país sostenía relaciones de css con menos de 40 países hacia el 2009, alcanzando en 2015 una cifra superior a los 70 (debido a su css con Asia y África), y en 2017 reportó css con un total de 66 países (APC-Colombia, 2018) ${ }^{13}$. En términos financieros la css que realiza el país a través de su agencia de cooperación, registra un monto cercano a los cop $\$ 140.000$ millones en el período 2009-2019 (ver Figura 2).
No obstante, esta cifra no incluye otros tipos de iniciativas de css realizadas al margen de la APC-Colombia. Es el caso, por ejemplo, de las acciones de css y Ст que lidera el Ministerio de Defensa Nacional en lo que este denomina la Diplomacia para la Seguridad y la Defensa. Según dicha cartera entre 2010 y 2017 se han capacitado 36.309 personas de 73 países en diferentes áreas como la lucha contra las drogas, el fortalecimiento de especialidades militares y policiales, entre otras (Ministerio de Defensa, 2018). Sobre esta cooperación realizada de forma independiente, advierte Tickner (2016) puede corresponder a metas institucionales y

Figura 1

Iniciativas bilaterales, regionales y triangulares de css colombiana.

Años 2009-2016

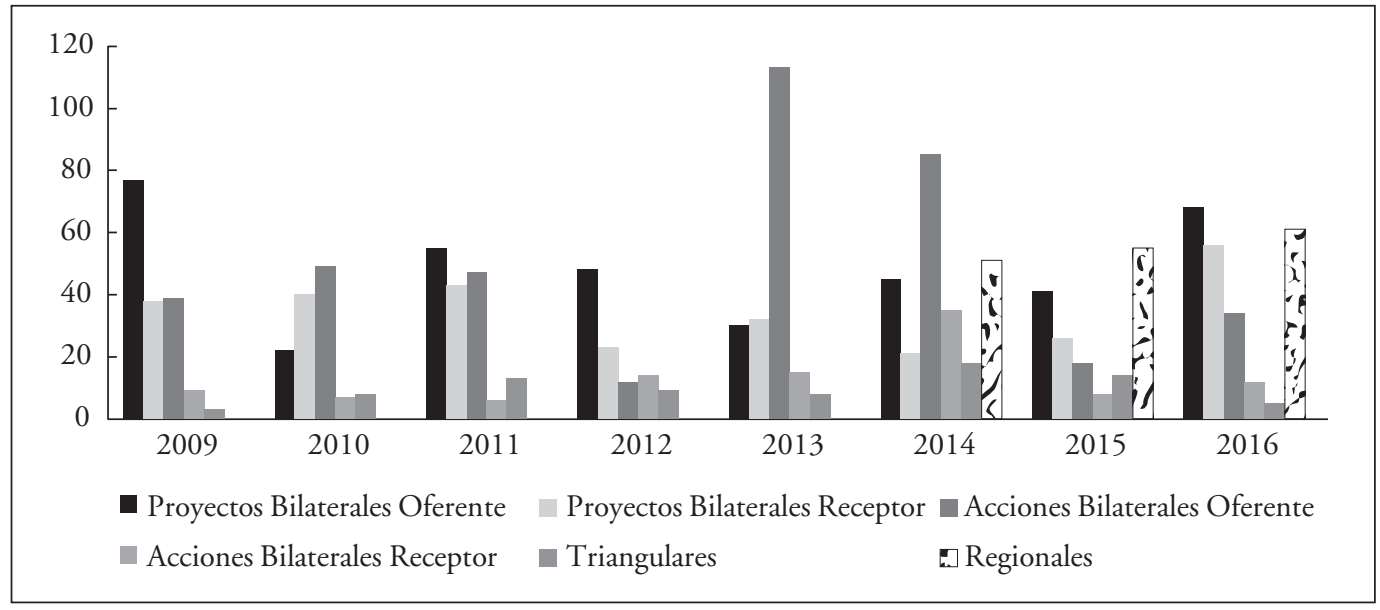

Fuente: Elaboración propia, 2019 a partir del Informe de css de Segib 2010-2018.

13 Según APC-Colombia (2019b), para el 2018 se encontraban en ejecución 126 proyectos de css con países de América Latina y el Caribe, África, Asia y Eurasia. De estos, 59 fueron aprobados en las nueve comisiones mixtas realizadas en dicho año. 
Figura 2

Presupuesto asignado para financiar la css colombiana (Focai e Inversión) Años 2009-2019 (En miles de millones cop\$)

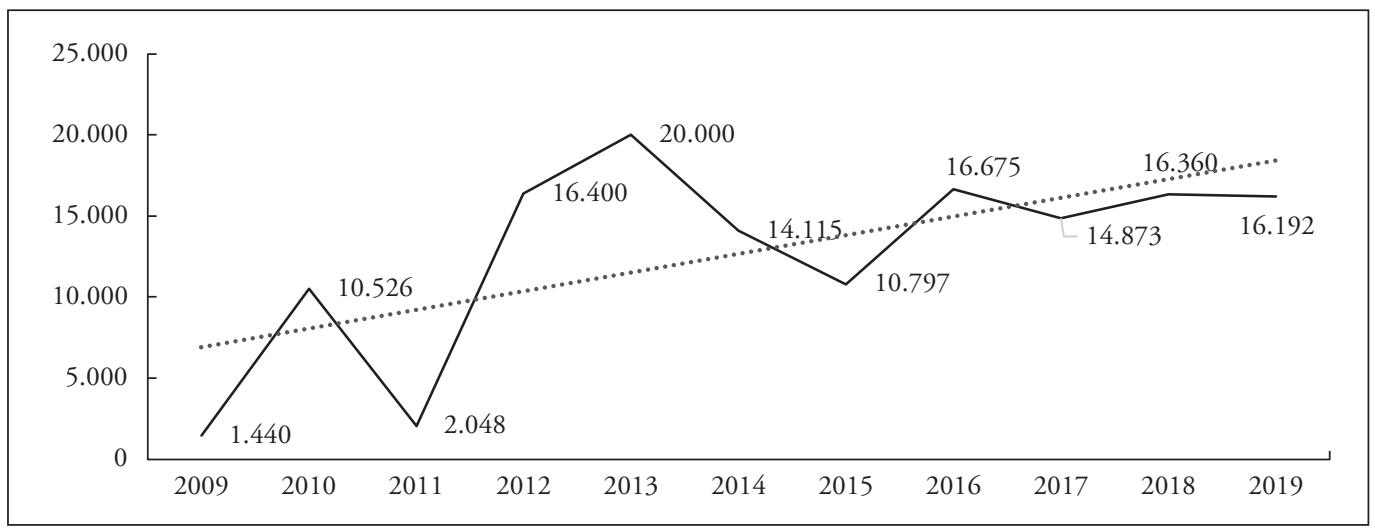

Fuente: Elaboración propia, 2019 a partir de Acción Social (2009), APC-Colombia (2012) y Ministerio de Hacienda 2011 a 2019, decretos anexos de liquidación del Presupuesto General de la Nación.

no necesariamente a las de la política exterior, pudiendo orientarse hacia logros diferentes e incluso contradictorios.

La información disponible permite evidenciar cómo la css es un elemento cada vez más relevante en la política exterior del país y como este tiene un papel cada vez más activo en la arena internacional como oferente de una css con alto enfoque técnico (Bergamaschi et al., 2017a). Se cuenta, además, con una apropiación presupuestal particularmente importante desde el 2012, y la cooperación regional, como lo evidencia la Segib, tiene ahora mayor dinamismo ${ }^{14}$.
Con la llegada de un nuevo gobierno nacional a finales de 2018 se espera profundizar lo ya recorrido y superar los retos aún pendientes de la css colombiana. De momento, las Bases del Plan Nacional de Desarrollo para el período 2018-2022 señalan la importancia de adelantar estrategias para fortalecer el papel de Colombia también como oferente de cooperación, atendiendo a los cambios en el acceso a recursos derivados de su nuevo estatus internacional, así como la diversificación temática y geográfica de la agenda exterior (DNP, 2019a).

14 Nivia-Ruiz y Prieto (2015) explican en detalle las cinco estrategias regionales de css (con el Caribe, Mesoamérica, África, Asia y de Seguridad Integral), aún vigentes, que lidera Colombia desde principios del s. XxI. 


\subsection{Cuantificación y agregación de valor en la css colombiana}

A los antecedentes hasta ahora señalados hay que adicionar uno más para llegar a este apartado con la perspectiva de análisis completa. En el 2015 el Gobierno Nacional lanzó la “Hoja de Ruta de la Cooperación Internacional 2015-2018” donde se definían los objetivos estratégicos de la AOD y la css para el país. La estrategia reconocía como uno de sus dos objetivos estratégicos, compartir conocimiento de alto valor para contribuir a la política exterior del país, al mismo tiempo que generar beneficios tangibles para el desarrollo de Colombia y los socios en la CSs у Cт (APC-Colombia, 2015a). Este sería el hecho de política que marcaría el inicio del camino actual de la cuantificación de la css colombiana.

A partir de entonces se iniciaron los debates y reflexiones sobre la forma más adecuada para avanzar en esta ruta que culminó, en parte, con el desarrollo de la "Caja de Herramientas de la css" en 2017, donde se establecen una serie de orientaciones y lineamientos sobre mecanismos, etapas e instrumentos de intercambio de conocimiento en la css, formatos de tipo técnico, logístico y de seguimiento. Adicionalmente presenta, por primera vez, el Modelo de Cuantificación y Agregación de Valor (MCAV) de la Css colombiana (APCColombia, 2017).
El primer componente del modelo hace referencia a la cuantificación de la css, para lo cual tomaron como base los ejercicios ya sistematizados por el pIFCss (2016) de Brasil, Chile y México, y se propone cuantificar para cada iniciativa de css colombiana los costos directos asociados a la misma, a saber: Boletos aéreos (Ba) incluyendo seguros médicos si aplica; Gastos de viaje (Gv); y Logística (Lg) ${ }^{15}$.

Adicionalmente, propone, por primera vez, cuantificar los costos indirectos asociados a los expertos/técnicos que comparten su conocimiento (llamados embajadores de conocimiento) en cada iniciativa de css para quienes calcula: Número de expertos participantes (n); Salario mensual (Sm); Salario diario que es el cociente resultante de dividir entre 20 el Sm (Sd); días de duración de la actividad más dos adicionales, considerados estos últimos de preparación técnica $(\mathbf{d}+\mathbf{2})$. En síntesis la fórmula de cuantificación propuesta es la siguiente:

\section{Cuantificación $=(B a+G v+L g)+\left(n^{*} S d^{*} d+2\right.$ \\ Costos directos + Costos indirectos}

Considerando un ejemplo sencillo en donde un funcionario colombiano se desplazara a Bolivia a desarrollar una agenda técnica, de 5 días de duración y APC-Colombia financiara su boleto aéreo a un costo de us $\$ 2.000$, en tanto que el Gobierno de Bolivia aportara los viáticos

15 Hasta el 2018, un porcentaje de los recursos del Focai se licitaba para que empresas especializadas en servicios de logística (alojamiento, alimentación, transporte, traducciones, etc.) los brindaran en el país, y fuera de este, en desarrollo de las iniciativas de CSs co-financiadas por APC-Colombia. Este rubro también puede incluir otro tipo de aportes de las entidades socias ejecutoras, como equipos, materiales, insumos, etc. 
para el delegado a us $\$ 80$ diarios. Los costos directos serían calculados de la siguiente forma:

$$
\begin{aligned}
& \mathrm{Ba}=\mathrm{us} \$ 2000 \\
& \mathrm{Gv}=\mathrm{us} \$ 400 \text { [80 valor diario } * 5 \text { días }] \\
& \mathrm{Lg}=\mathrm{us} \$ 0 \text { [No aplica] }
\end{aligned}
$$

Ahora bien, para establecer el valor de los salarios de los expertos, y considerando el complejo universo de escalas, niveles y rangos salariales que existen en el sector público, el MCAV propone eliminar esta posible distorsión a partir del uso de un instrumento de referencia del Departamento Nacional de Planeación (DNP) que estima los honorarios mensuales de las personas combinando su formación académica (desde la educación básica hasta la superior) con su experiencia ${ }^{16}$.

En este caso, y para continuar con el ejemplo, si el experto colombiano (embajador de conocimiento) fuese un profesional con posgrado a nivel de especialización y 22 meses de experiencia relacionada, el citado instrumento del DNP lo ubica en un único nivel de ingreso (cop\$7.017.615) que deberá ser convertido a dólares estadounidenses. En el caso del ejemplo, el cálculo de los costos indirectos, sería así:

$$
\begin{aligned}
& \mathrm{n}=1 \quad[1 \text { experto }] \\
& \mathrm{Sd}=\mathrm{us} \$ 106[(7.017 .615 / 20) / \mathrm{trm}] \\
& \mathrm{d}+2=7 \quad[5 \text { días }+2 \text { días de preparación }]
\end{aligned}
$$

En síntesis la cuantificación total de esta iniciativa sería:

$$
\begin{aligned}
\text { Cuantificación } & =(2.000+400+0)+\left(1^{*} 106^{*} 7\right) \\
& =\text { Us\$ } 3.142
\end{aligned}
$$

Ahora bien, a diferencia de las otras experiencias consideradas por Colombia, el componente de cuantificación del MCAV propone realizar esta medición tanto en la fase de formulación del proyecto (y sus respectivas actividades) como en la fase de culminación de este, lo cual permitirá comparar los dos momentos con información financiera real y contrastar la ejecución versus la formulación en términos de costos directos e indirectos, permitiendo la mejora en procesos de planeación posteriores.

En segunda instancia, el concepto novedoso que incorpora el MCAV es el de la agregación de valor, como el elemento a través del cual el país busca valorar las contribuciones que efectivamente realizan las iniciativas de css en el desarrollo del país y de los países socios con los que se implementa. Para tales efectos, este segundo componente de agregación de valor define cinco categorías con sus respectivos indicadores y alcance esperado, en los cuales se busca establecer cómo la implementación de iniciativas de css en realidad agrega valor y contribuye al desarrollo, en términos cualitativos más que monetarios (ver Tabla 3).

\footnotetext{
16 Se hace referencia a la "Tabla de honorarios de contratos de prestación de servicios profesionales y de apoyo a la gestión” del DNP que fija los valores y criterios, así como las respectivas equivalencias.
} 
Tabla 3

\section{Componente de agregación de valor del modelo:}

Categorías, indicadores, alcance y valoración

\begin{tabular}{|c|c|c|c|}
\hline Categorías & Indicadores & Alcance & Valor final \\
\hline \multirow{3}{*}{$\begin{array}{l}\text { CONOCIMIENTO. Generación de } \\
\text { nuevos conocimientos aplicables } \\
\text { en algún ámbito del desarrollo } \\
\text { que favorecen cambios } \\
\text { constatables. }\end{array}$} & $\begin{array}{l}\text { 1.1. Técnicas, métodos, saberes, metodologías } \\
\text { desarrolladas o mejoradas. }\end{array}$ & \multirow{3}{*}{$\begin{array}{l}\text { Conocer } \\
\text { Apropiar } \\
\text { Aplicar } \\
\text { Replicar }\end{array}$} & \multirow{3}{*}{ Mínimo: 0} \\
\hline & $\begin{array}{l}\text { 1.2. Participantes que adquieren mayores capa- } \\
\text { cidades, competencias o desarrollan habilidades } \\
\text { ya existentes. }\end{array}$ & & \\
\hline & 1.3. Productos de aprendizaje elaborados. & & \\
\hline \multirow{3}{*}{$\begin{array}{l}\text { RELACIONAL. Generación o } \\
\text { afianzamiento de relaciones y } \\
\text { sinergias, que se transforman } \\
\text { en redes de trabajo, modelos } \\
\text { colaborativos, entre otros. }\end{array}$} & $\begin{array}{l}\text { 2.1. Técnicos/expertos de instituciones de los } \\
\text { países que entran en contacto y se relacionan } \\
\text { con sus homólogos. }\end{array}$ & \multirow{3}{*}{$\begin{array}{l}\text { Informal } \\
\text { Inactivo } \\
\text { Informal activo } \\
\text { Formal inactivo } \\
\text { Formal activo }\end{array}$} & \multirow{3}{*}{$\begin{array}{l}\text { Mínimo: } 0 \\
\text { Máximo: } 9\end{array}$} \\
\hline & 2.2. Redes establecidas. & & \\
\hline & $\begin{array}{l}\text { 2.3. Comunidades de conocimiento y apren- } \\
\text { dizaje. }\end{array}$ & & \\
\hline \multirow{3}{*}{$\begin{array}{l}\text { VISIBILIDAD. Identificación y } \\
\text { visibilización de los resultados } \\
\text { de aprendizaje obtenidos con el } \\
\text { proyecto. }\end{array}$} & $\begin{array}{l}\text { 3.1. Proyecto difundido en medios de comuni- } \\
\text { cación propios (físicos o digitales) de las institu- } \\
\text { ciones socias del proyecto. }\end{array}$ & \multirow{3}{*}{$\begin{array}{l}\text { Difundido } \\
\text { No difundido }\end{array}$} & \multirow{3}{*}{$\begin{array}{l}\text { Mínimo: } 0 \\
\text { Máximo: } 9\end{array}$} \\
\hline & $\begin{array}{l}\text { 3.2. Proyecto difundido en medios de comu- } \\
\text { nicación (físicos o digitales) del(los) país(es). }\end{array}$ & & \\
\hline & $\begin{array}{l}\text { 3.3. Proyecto difundido en medios de comuni- } \\
\text { cación especializados. }\end{array}$ & & \\
\hline $\begin{array}{l}\text { ALINEACIÓN CON LOS ODS. } \\
\text { Contribución concreta a } \\
\text { visibilizar y mejorar prácticas } \\
\text { asociadas con el cumplimiento } \\
\text { de los oDs. }\end{array}$ & $\begin{array}{l}\text { 4.1. ODS a los cuales contribuye directamente } \\
\text { el proyecto. }\end{array}$ & $\begin{array}{l}\text { Alineado } \\
\text { No alineado }\end{array}$ & $\begin{array}{l}\text { Mínimo: } 0 \\
\text { Máximo: } 9\end{array}$ \\
\hline $\begin{array}{l}\text { ENFOQUE DIFERENCIAL. Favorecer } \\
\text { la participación de la mujer y de } \\
\text { grupos étnicos. }\end{array}$ & $\begin{array}{l}\text { 5.1. Porcentaje de participación de mujeres y/o } \\
\text { grupos étnicos como beneficiarios directos en el } \\
\text { proyecto de los países participantes. }\end{array}$ & $\begin{array}{l}\% \text { de } \\
\text { participación }\end{array}$ & $\begin{array}{l}\text { Mínimo: } 0 \\
\text { Máximo: } 9\end{array}$ \\
\hline
\end{tabular}

Fuente: Elaboración propia, 2019 con base en APC-Colombia (2017).

El componente de agregación de valor, según el modelo propuesto por APC-Colombia, se debe diligenciar en tres momentos. Al formular y al concluir el proyecto (al igual que el componente de cuantificación), pero además se realiza al medio término de ejecución de la iniciativa, con el objetivo de establecer los avances en cada una de sus categorías e indicadores, y posibilitar acciones de mejora durante la ejecución en caso de ser necesario.

Al finalizar la iniciativa, las entidades técnicas ejecutoras junto a las respectivas agencias 
de cooperación, o quien haga sus veces en los países involucrados, asignarán una valoración final para cada uno de los indicadores de forma que cada categoría pueda alcanzar un puntaje que variará entre 0-9. Esta valoración debe encontrarse soportada con las evidencias técnicas que demuestren realmente los logros en términos de desarrollo alcanzados por la iniciativa implementada. La agregación de valor, dada su complejidad en términos de variables, momentos, puntajes, se representa visualmente mediante un gráfico radial, como se verá en el siguiente apartado (ver Figura 3).

Por último, el MCAV permite generar información adicional respecto a las iniciativas ejecutadas como el número total de instrumentos de intercambio de conocimiento ${ }^{17}$ utilizados, el total de expertos movilizados, la sumatoria total de los días efectivos de css entre pares, entre otros.

Esta propuesta metodológica liderada por APC-Colombia ha sido socializada y compartida con algunos países de la región, en el marco del pifCss, con miras a su implementación conjunta, algo que también se constituye en un elemento innovador a la vez que retador. El MCAV busca convertirse en el instrumento de medición referente de la css colombiana que reconozca, como lo señala Domínguez (2018), la creación y la gestión del conocimiento como dimensiones sustantivas y prioritarias en la evaluación de la css.

\subsubsection{El MCAv: Un ejercicio real de aplicación ${ }^{18}$}

Los gobiernos de Colombia y Bolivia ejecutaron en la vigencia 2016-2019 una iniciativa de css orientada al fortalecimiento de las capacidades productivas del cultivo de la quinua en cuatro municipios del Departamento de Boyacá (Colombia), a partir de las capacidades y experiencia del reconocido Centro Internacional de la Quinua (CIQ), creado en Bolivia, país que se posiciona entre los principales productores a nivel internacional, con más de 50 millones de toneladas por año (CIQ, 2016), y que cuenta con el banco de germoplasma más importante a nivel mundial con más de 2.500 accesiones de quinua (Iica, 2015).

El proyecto de css en mención ejecutó cinco misiones de intercambio en Bolivia y Colombia, junto a jornadas de trabajo virtuales entre el CIQ, la Gobernación de Boyacá y directamente representantes de cuatro asociaciones de productores de quinua del citado departamento colombiano. Una vez concluida la ejecución de la iniciativa bilateral y realizado el ejercicio de cuantificación (ver Tabla 4) y agregación de valor (ver Tabla 5) con la participación de las entidades técnicas ejecutoras y

17 La citada Caja de Herramientas propone trece modalidades que posibilitan iniciativas de css con intercambio efectivo de conocimiento. Precisa cuándo se recomiendan y sugerencias metodológicas para implementarlas.

18 Para la realización de este apartado el autor agradece a APC-Colombia permitir la difusión de la información y los resultados que ilustran la utilización del MCAV de la css colombiana con un caso real. 
las entidades responsables de la css de ambos países, se muestran los resultados del ejercicio técnico:

\section{Tabla 4}

Resultados componente cuantificación: Proyecto quinua (en us\$)

\begin{tabular}{|l|c|c|c|}
\hline \multicolumn{1}{|c|}{ Costos } & $\begin{array}{c}\text { Cuantifica- } \\
\text { ción inicial }\end{array}$ & $\begin{array}{c}\text { Cuantifica- } \\
\text { ción final }\end{array}$ & $\begin{array}{c}\text { Varia- } \\
\text { ción }\end{array}$ \\
\hline $\begin{array}{l}\text { Boletos } \\
\text { aéreos }\end{array}$ & 10.104 & 7.680 & $-24 \%$ \\
\hline $\begin{array}{l}\text { Gastos de } \\
\text { viaje }\end{array}$ & 10.234 & 8.047 & $-21,3 \%$ \\
\hline Logística & 6.500 & 6.873 & $+5,7 \%$ \\
\hline $\begin{array}{l}\text { Subtotal } \\
\text { costos } \\
\text { directos }\end{array}$ & 26.838 & 22.601 & $-15,7 \%$ \\
\hline $\begin{array}{l}\text { Subtotal } \\
\text { costos } \\
\text { indirectos }\end{array}$ & 4.750 & 8.139 & $+71,3 \%$ \\
\hline $\begin{array}{l}\text { Total } \\
\text { cuantificación }\end{array}$ & 31.588 & 30.740 & $-2,6 \%$ \\
\hline
\end{tabular}

Fuente: Elaboración propia, 2019, a partir de datos de APC-Colombia.

Estos resultados permiten diversos análisis y reflexiones, acá se destacan solo tres elementos considerados innovadores. Por primera vez, será posible comparar en una sola herramienta, la ejecución financiera final de cada iniciativa de css con la estimación financiera inicial, lo cual con seguridad contribuirá a ejercicios de formulación presupuestal cada vez más precisos, y que le permitan a APC-Colombia hacer un uso más eficiente de los recursos de la css.

En segundo lugar, permite, también por primera vez, contar con información precisa sobre los costos indirectos de la css en la que
Colombia participa. A la fecha, el país únicamente ha logrado reportar los costos directos de la css que se financian a través del Focai. Por último, toda esta información es valiosa no solo para los reportes que el país presenta, sino que servirá para motivar y alentar a las propias entidades ejecutoras de las iniciativas, a contar con información confiable sobre la css en la cual participan, y que por sí solas no todas estarían en la capacidad de generar.

El segundo componente del MCAV permite estimar los resultados concretos cualitativos y cuantitativos (no financieros) que la implementación de la css ofrece y pone a disposición del mundo del Sur Global, y sobre el cual vale la pena destacar lo siguiente.

En primer lugar, su aplicación requiere de una capacidad técnica importante por parte de los países y las entidades involucradas para obtener los resultados propuestos. Adicionalmente, se hace evidente que pueden surgir y existir resultados en dimensiones y categorías que el modelo no aborda, y que, con seguridad, no es posible reflejar al menos en esta versión del mismo. Por último, existe la posibilidad que no todas las iniciativas de css tendrán resultados tan positivos como el ejemplo que ilustra este escrito, y ello pueda limitar los incentivos que tengan las entidades para profundizar el uso de este tipo de herramientas novedosas.

El MCAV se convierte en una apuesta que pretende resignificar el valor que tiene la css para Colombia. Intenta, por una parte, cuantificar los costos directos tradicionales, tal y como sucede en algunos países de la región y, asimismo, incluye, por primera vez, los costos indirectos asociados a las horas dedicadas por los llamados embajadores de conocimiento. Su 
Tabla 5

Resultados componente agregación de valor: Proyecto quinua

\begin{tabular}{|c|c|c|c|}
\hline Categorías & Logros según indicadores & Alcance & Valor final \\
\hline \multirow[t]{3}{*}{ Conocimiento } & $\begin{array}{l}\text { 1.1. Técnica del cultivo de la quinua fortalecida en } 5 \mathrm{mu}- \\
\text { nicipios a partir de las capacidades de Bolivia. }\end{array}$ & Aplicado & \multirow{3}{*}{9} \\
\hline & $\begin{array}{l}\text { 1.2. Cinco asociaciones de productores beneficiadas. Vin- } \\
\text { culación de una universidad en trabajos de campo e inves- } \\
\text { tigación sobre quinua. }\end{array}$ & Aplicado & \\
\hline & $\begin{array}{l}\text { 1.3. Elaboración y distribución de una cartilla para produc- } \\
\text { tores de quinua ( } 1000 \text { ejemplares). }\end{array}$ & Aplicado & \\
\hline \multirow[t]{3}{*}{ Relacional } & $\begin{array}{l}\text { 2.1. Más de } 200 \text { personas vinculadas al proyecto entre } \\
\text { productores de quinua en Colombia y expertos del cIQ. }\end{array}$ & Informal - activo & \multirow{3}{*}{6} \\
\hline & $\begin{array}{l}\text { 2.2. No registra la creación de nuevas redes, aunque forta- } \\
\text { lece las existentes en el orden local. }\end{array}$ & Informal - activo & \\
\hline & $\begin{array}{l}\text { 2.3. Nueva comunidad aprendizaje posibilitada entre el } \\
\text { CIQ de Bolivia y una universidad en Boyacá (pasantías e } \\
\text { investigación). }\end{array}$ & $\begin{array}{l}\text { Formal - activo } \\
\text { (en proceso) }\end{array}$ & \\
\hline \multirow[t]{3}{*}{ Visibilidad } & $\begin{array}{l}\text { 3.1. Elaboración de más de } 15 \text { piezas de difusión sobre } \\
\text { los resultados de la iniciativa por parte de las entidades } \\
\text { ejecutoras. }\end{array}$ & Difundido & \multirow{3}{*}{8} \\
\hline & $\begin{array}{l}\text { 3.2. Elaboración de } 3 \text { piezas de difusión sobre los resultados } \\
\text { de la iniciativa en medios locales y departamentales. }\end{array}$ & Difundido & \\
\hline & $\begin{array}{l}\text { 3.3. Elaboración de } 20 \text { programas radiales sobre las técnicas } \\
\text { del cultivo de la quinua para productores del Departamento. }\end{array}$ & Difundido & \\
\hline $\begin{array}{l}\text { Alineación con } \\
\text { los oDs }\end{array}$ & 4.1. Alineación y contribución a 6 oDs. & Alineado & 9 \\
\hline $\begin{array}{l}\text { Enfoque } \\
\text { diferencial }\end{array}$ & $\begin{array}{l}\text { 5.1. El total de beneficiarios del proyecto, principalmente } \\
\text { en las asociaciones de productores de quinua, son mujeres. }\end{array}$ & $60 \%$ & 9 \\
\hline
\end{tabular}

Fuente: Elaboración propia, 2019, a partir de datos de APC-Colombia.

componente de agregación de valor, por otra parte, supone una propuesta de valorización de la css en términos de contribuciones al desarrollo, que aporta a lo que el PIFCSs (2013) ya reseñaba como uno de los aspectos que más interés despierta y sobre el cual la región propone trabajar en el mediano plazo: la estimación del valor de la css.
No cabe duda alguna que esta es una generación digital en donde la información será siempre un elemento fundamental para implementar cada vez mejores políticas en materia de gestión pública. Como bien lo señala Oszlak (2013) al hacer referencia al concepto de información, corresponde distinguir entre datos, información y conocimiento. Solo la conversión de datos en información y de estos 
Figura 3

Representación final del componente de agregación de valor: Proyecto quinua

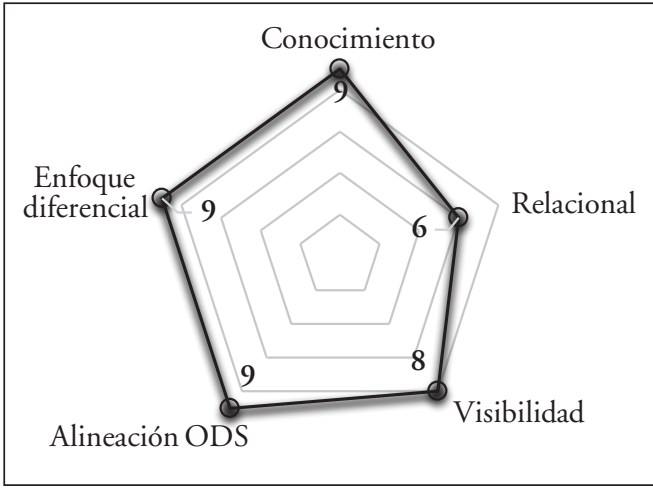

Fuente: Elaboración propia, 2019, a partir de datos de APC-Colombia.

en conocimiento permitirá generar fundamentos técnicos y políticos para elegir cursos de acción. Este parece ser el camino en el cual Colombia busca profesionalizar cada vez más la css que ofrece y recibe.

\section{CONCLUSIONES}

El país aún enfrenta importantes retos para elaborar, consolidar y difundir los datos e información que produce sobre su css. Los cambios institucionales vividos y la falta de memoria institucional que ello provoca, junto a la falta de uniformidad con que son presentadas las cifras año a año, y las diversas fuentes que reportan información que no necesariamente coinciden entre sí, generan dificultades metodológicas para este tipo de escritos que buscan comprender y analizar la css colombiana.
Resulta también claro que la mayor proliferación de cifras, hechos, información y reportes estén asociados a los flujos de AOD, antes que a las dinámicas de la css. Esto puede resultar al menos comprensible para un país que aún recibe más de us\$ 400 millones anuales en AOD, y que hasta hace muy poco dimensiona su rol dual como oferente y receptor, con una apuesta más estructurada en términos de su css.

No obstante, es evidente que existe un claro interés en dar cada vez mayor rigor técnico y metodológico a la css que se ejecuta. La Caja de Herramientas constituye una muestra de un esfuerzo técnico juicioso que, si bien no podrá garantizar al 100\% la exitosa ejecución de las iniciativas de css, por lo menos busca reducir en gran medida las externalidades negativas y factores de riesgo que se deben considerar al momento de implementar iniciativas de css.

La nueva apuesta del MCAV, va muy orientada a lo señalado por el Paba+40 en el sentido de elaborar sistemas propios que mejoren la recopilación, coordinación y difusión de información y datos en términos de calidad, efectos y evaluación de la css, y resulta pionera según los tres tipos de enfoques de acción y discusión vigentes que se describieron líneas arriba sobre la cuantificación de la css.

De igual forma, la propuesta colombiana se considera una experiencia que puede contribuir a los recientes debates sobre la medición de la css, a cuarenta años del Paba, al incorporar y rescatar elementos ya desarrollados sobre cuantificación monetaria de la css, así como aportando elementos que pueden dar luz al camino de la valorización de la css. Esta 
tarea supone retos y desafíos que brevemente tratarán de ser resumidos.

$\mathrm{Al}$ ser una herramienta novedosa requiere, primero, un alto nivel de apropiación por parte de la propia APC-Colombia para lograr que sea utilizada adecuadamente en todas las iniciativas de css que la agencia lidera con un número importante de entidades nacionales, territoriales y no gubernamentales. El primer ejercicio de difusión, pedagogía y orientación deberá adelantarse en casa, lo cual genera ya un desafío a las actuales capacidades con que cuenta APC-Colombia para la gestión de la css.

De igual forma, requiere que las entidades colombianas y en los países socios, encuentren los incentivos suficientes, como ya se ha indicado, para compartir la información financiera, cuantitativa y cualitativa sobre la implementación de las iniciativas de css. Dado que el MCAV es de uso interno de la Agencia y no permite generar acceso a terceros para el ingreso de datos, deberá tener un uso muy estandarizado en el registro de la información. Se espera que con la entrada en funcionamiento del sistema de información Cíclope esto pueda verse resuelto de una mejor forma.

Es claro que el alcance del MCva está limitado, de momento, a la css que gestiona y co-financia APC-Colombia. No obstante, como se evidenció líneas arriba, esta es apenas una parte del universo de la css que realizan otras entidades del orden nacional, regional y local. Por otra parte, vale la pena reflexionar sobre la utilidad de vincular al Instituto Nacional de Estadística y articular el trabajo que ambas entidades realizan en la generación de cifras, información, indicadores y conocimiento cada vez más apropiados para analizar y medir la css colombiana.

Asimismo, con la implementación del modelo podrán salir a la luz un número importante de hechos, evidencias, costos, indicadores que escapan al alcance de este, y sobre los cuales se plantean tres posibles opciones. Primero, no incluirlos de momento con el ánimo de no alterar la herramienta; incluirlos como nota al pie aunque sin modificar lo ya construido; o bien proponer ajustes estructurales al modelo para incorporar tantos ajustes como sea necesario, lo cual puede resultar un riesgo metodológico en sí mismo.

Colombia ha iniciado, como otros países, el tránsito de un camino para realizar la medición cuantitativa y de agregación de valor de su css, en medio de las discusiones y debates que aún persisten sobre el tema, y que son útiles para los tomadores de decisiones en los diferentes países. Es importante que estas nuevas herramientas queden plasmadas en los nuevos instrumentos de política de la cooperación internacional en el país, de forma que logren ser sostenibles y generar resultados significativos con el tiempo.

El hecho de que el modelo plantee un ejercicio conjunto entre las entidades de cooperación y las instituciones técnicas ejecutoras; que involucre a las contrapartes de los países involucrados; que se realice en tres momentos durante la ejecución de una iniciativa; y que considere elementos más allá de lo monetario, son solo algunos de los elementos innovadores y mayores desafíos sobre los cuales se espera tener mayores evidencias en el mediano plazo. 


\section{REFERENCIAS}

Acción Social. (2009). Informe de Gestión de la Cooperación Internacional en 2009. Bogotá.

Acción Social. (2008). Cuarenta años de la institucionalidad de la cooperación internacional en Colombia. Bogotá.

Acción Social. (2005). Estrategia de Cooperación Internacional. Cartagena.

Acci. (2002). Informe de Actividades 2001-2002. Bogotá.

APC-Colombia. (2019a). Informe de Gestión 2018. Bogotá.

APC-Colombia. (2019b). Rendición de Cuentas 2018. Bogotá.

APC-Colombia. (2018). Informe de Gestión 2017. Bogotá.

APC-Colombia. (2017). Caja de Herramientas de la css Colombia. Bogotá.

APC-Colombia. (2015a). Hoja de Ruta de la Cooperación Internacional 2015-2018. Bogotá.

APC-Colombia. (2015b). Informe de Gestión. Vigencia 2014. Bogotá.

APC-Colombia. (2012). Informe de Gestión de la Cooperación en Colombia. Bogotá.

Ayllón, B.; Ojeda, T. y Bancet, A. (2013). La css en la gobernanza del desarrollo: Nuevas configuraciones de la arquitectura de la ayuda. Documento de Trabajo, No. 27. UIDC-UCM.

Ayllón, B. (2015). La css en América Latina y el Caribe. De una época dorada a una fase incierta. Anuario de Integración, 11, 135-170.

Bergamaschi, I.; García, J. y Santacruz, C. (2017a). Colombia como oferente y receptor de cooperación internacional: apropiación, liderazgo y dualidad. En Tickner, A. Bitar, S. (Eds.), Nuevos enfoques para el estudio de las relaciones internacionales de Colombia. Uniandes, pp. 331-361.
Bergamaschi, I.; Tickner, A. y Durán, J. (2017b). Going South to Reach the North? The case of Colombia. En Bergamaschi, I. (Ed.), South-South Cooperation Beyond the Myths. Rising Donors, New Aid Practices?, pp. 245-270.

Besherati, N. y MacFeely, S. (2019). Defining and Quantifying South-South Cooperation. Unctad Research Paper, No. 30.

Bracho, G. (2018). Towards a Common Definition of ssc: Bringing Together the Spirit Of Bandung and Buenos Aires. Development Cooperation Review, 1(6), 9-13.

BRICs Policy Center y Articulação Sul. (2017). Paths for Developing SSC Monitoring and Evaluation Systems. Brasilia.

Centro Internacional de la Quinua. (2016). Bolivia se consolida como sede oficial del Centro Internacional de la Quinua. Julio.

Departamento Nacional de Planeación. (2019a). Bases del Plan Nacional de Desarrollo 2018-2022. Bogotá.

Departamento Nacional de Planeación. (2019b). Tabla de honorarios de contratos de prestación de servicios profesionales y de apoyo a la gestión. Bogotá.

Departamento Nacional de Planeación. (1995). Conpes 2768. Política Nacional de Cooperación Internacional. Bogotá.

Departamento Nacional de Planeación. (1982). Decreto 2157. No. 36065. Bogotá, 19 de julio.

Departamento Nacional de Planeación. (1958). Ley 19. No. 29.835. Bogotá, 9 de diciembre.

Di Ciommo, M. (2017). Approaches to measuring and monitoring South-South cooperation. Development Initiatives.

Domínguez, R. (2018). Hacia un régimen internacional de css: últimos avances sobre el monitoreo y la evaluación. Estado Abierto, 2(2), pp. 49-108. 
Grupo de Tarea en Cooperación Sur-Sur-GT-CSS. (2010). La cSS en el contexto de la eficacia de la ayuda. 110 historias de casos de socios en CSS y CT. Bogotá.

Guáqueta. (1995). La cooperación técnica en Colombia como una herramienta estratégica de política exterior. Colombia Internacional, 30, pp. 3-15.

Henao, J. (1991). La Cooperación Internacional al Desarrollo en Colombia. Bogotá: Centro de Investigación y Educación Popular.

Huitrón, A. (2016). La css y el reto de su cuantificación, evaluación y valoración. Revista Iberoamericana de Estudios de Desarrollo, 5(1), pp. 88-122.

Iica. (2015). Producción y mercado de la quinua en Bolivia. La Paz.

Ilpes. (1984). Simposio de Alto Nivel sobre Cooperación Técnica Internacional. Proyecto de Informe de Relatoría. Ciudad de México.

Lazo, C. (2018). La Cooperación Internacional de Chile a 40 años del Paba. Revista Española de Desarrollo y Cooperación, 43, pp. 85-94.

Lopes, M. (2017). La cuantificación de la css y sus consecuencias para la política exterior de los países en desarrollo. Centro del Sur, No. 41.

Mideplan. (2018). Guía para determinar el valor de las contrapartidas institucionales en los proyectos de cooperación internacional. San José.

Ministerio de Defensa Nacional. (2018). Memorias al Congreso 2016-2017. Bogotá.

Ministerio de Hacienda. (2019). Anexo Decreto del Presupuesto General de la Nación. Bogotá.

Naciones Unidas. (2018). Estado de la css. Informe del Secretario General. A/73/321.

Nivia-Ruiz, F. (2015). Colombia y la css regional: Una experiencia de innovación y posicionamiento internacional. Revista Española de Desarrollo y Cooperación, 36, pp. 111-122.
Nivia-Ruiz, F. (2009). La CSS en América Latina y el Caribe. Aproximación a un estado del arte. En Escuela Latinoamericana de Cooperación y Desarrollo (Ed.), Cooperación Euroandina y Sur-Sur en América Latina, pp. 125-220.

OECD. (2018). Development Co-operation Report 2018. Joining forces to leave no one behind. Paris.

Oszlak, O. (2013). Gobierno abierto: hacia un nuevo paradigma de gestión pública. Red Gealc. Septiembre.

Pérez, J. y Huitrón, A. (2018). Debate sobre la medición de la css: consideraciones para la cooperación mexicana. Oxfam. Julio.

PIFCSs. (2016). Valorización de la CSS. Estudios de Caso: Brasil, Chile y México. Documento de Trabajo, No. 10.

PIFCSS. (2013). Generando indicadores para la CSS. Documento de Trabajo, No. 4.

PNUD. (2016). Scaling-up SSC for sustainable development. Nueva York.

Prado, J. (2016). La gobernanza de la css. En Soares de Lima, M.; Milani, C. y Echart, E. (Ed.), css, politica exterior y de modelos de desarrollo en América Latina. Clacso.

Ramírez, S. (2005). Encrucijadas de la cooperación internacional en Colombia. En Ardila, M.; Cardona, D. y Socorro, R. (Ed.), Colombia y su politica exterior en els. XXI. Fescol.

Sanín, M. (2010). La css en Colombia. En Ayllón, B. y Surasky, J. (Coord.), La CSS en Latinoamérica. Utopia y Realidad. Madrid, pp. 103-123.

Segib. (2018a). Informe de la css en Iberoamérica 2018. Madrid.

Segib. (2018b). Una década de Cooperación Sur-Sur en Iberoamérica 2007-2017. España.

Surasky, J. y Ayllón, B. (2018). 40 años no es nada: del Paba a la Segunda Conferencia de Alto Nivel de 
las Naciones Unidas sobre la css. Revista Española de Desarrollo y Cooperación, 43, pp. 37-51.

Surasky, J. (2018). Seguimiento a la css (mayo-septiembre). Revista Española de Desarrollo y Cooperación, 43, pp. 199-209.

Tickner, A. (2016). Exportación de la seguridad y política exterior de Colombia. Análisis. Friedrich Ebert Stiftung.

Tomassini, L. (1976). El proceso de cooperación técnica entre países en desarrollo. Integración Latinoamericana, 1(4), pp. 26-32.
Xalma, C. (2013). El renovado auge de la css: La experiencia iberoamericana. Integración y Comercio, 36, pp. 29-42.

Yu, V. (2019). Key issues for BAPA+40: ssC and the bapa+40 subthemes. South Centre. Research Paper, No. 91. 